is prescribed during the existence of febrile excitement, the dose, to be efficacious, must be large, and the impression on the disease sudden and overwhelming.

An auxiliary, too, is also required in such cases: twentyfour grains of quinine and twenty grains of calomel, in one dose, is the most powerful resolvent of fever. One or two such doses, with an interval of six hours, and followed by a castor oil purgative, are generally sufficient; but I have prescribed six such doses with efficacy, and I recollect no instance of ptyalism occurring when this treatment was required and adopted, and sometimes there is but mild cinchonism. An intolerance of quinine, or early and intense cinchonism, in such cases, is one of the worst prognostics.

In the treatment of simple intermittent fever, or its relapses, calomel is rarely, if ever, prescribed by the writer. Sulphate and carbonate of magnesia mixture, or sulphate of magnesia and tartrate of antimony mixture, as a purgative during the hot stage, (if needed,) or fifteen drops of solution of acetate of morphine, with a drachm of sweet spirits of nitre, if there is much suffering from muscular pains, headach, or emesis and retching, will speedily relieve the paroxysm; and followed by quinine, in combination with purgative doses of rhubarb, will fulfil all the indications for the intermission. But when a European or North American, probably not long from a cold climate, and during the prevalence of malignant disease, is attacked by fever, and shows to the quick and practised eye alarming indications, no fear of the injurious after-effects of the mercurial will have weight to withhold the resolvent dose of calomel and quinine. In cases threatening danger to life only need it be used, and I know of no instance wherein the slightest untoward result has been experienced from its use.

The combination of quinine with tartar-emetic in pneumonic and bronchitic complications of intermittent is eminently successful. The forces which disturb the remedial power of quinine in fever are chiefly inflammatory and congestive complications, or a loaded condition of the alimentary canal. These must be obviated by appropriate treatment, and the disease rendered as simple or idiopathic as possible, concurrent with the use of quinine. Thus arteriotomy may frequently be required in continued, remittent, or yellow fever; and in intermittent, with tenderness over spleen, a blister may be required, as an auxiliary to cinchonism.

There is a form of continued, or irregular remittent fever, occurring chiefly in children or adolescents, in which generally no local cause can be discovered, but which is often imputed to worms; but give what anthelmintics you will, no worms may be passed; hence here they are popularly called "stubborn worms." This fever may continue for a week or a fortnight without any contamination of the blood or loss of vital cohesion, and probably depends on intestinal irritation. Danger in these cases chiefly arises from the supervention of some lesion, induced by the long-continued and excessive heat and violent action of the heart, or sympathetic irritation of the brain. In these cases I use quinine, with immediate and signal efficacy, in the following manner:-

The patient is put into a bath, and the cold affusion is applied till the pulse becomes small, and nearly extinct, at the wrist, and the skin cold. He then, while in the bath, gets his dose of quinine, (two or three grains,) and is returned to bed without being dried. The bath and the dose of quinine are continued hourly as long as the skin persists warm, when the hourly dose of quinine is due. After five or six baths the skin generally becomes permanently cool, and then the quinine is pushed on to cinchonism, alone, and without the bath. This mode of making an intermission in a continued fever I have never found attended with unpleasant or dangerous consequences, and it will generally subdue the fever after every other method has been tried in vain.

In fever of doubtful origin, and where latent inflammation is suspected, I have frequently used a small cantharides blister as a test: in fact, $I$ never like to pass the blistered surface of a patient without inspecting it, its revelations are often so interesting and important. If, instead of the usual vesication of thin serum and cuticle, the vesication is a bladder of fibrinous coagulum, or suety in consistence, inflammatory action is going on, probably in the neighbourhood of the part, and tartar-emetic, or such-like combinations, are indicated.

Relapses in intermittents have their determinate periods, the day from the last attack being generally some multiple of seven.

The usual day of relapse among the acclimatized of this colony is the fourteenth or twenty-eighth.

After one or two relapses, the law of each individual case can be ascertained by each patient.
The prophylactic which I have adopted with great success, and in my own person first, many years ago, is as follows:-

Two days before the anticipated relapse, three grains of quinine, to be taken thrice daily for four days; and after a similar relapse interval, the quinine to be again taken in the same manner; and so on, repeated three or four times successively. The disease is eradicated completely by thus baffling the relapse.

Georgetown, British Guiana, Sept. 1847.

REPORT OF A
DEATH FROM INTERNAL STRANGULATION OE
THE SMALL INTESTINES,

WITH REMARKS ON THE PKOPRIETY OF AN OPERATION FOR RELIET OF THE DISEASE.

By ISAAC MASSEX, M.D., \&c., Nottingham.

The question of the propriety of making large sections through the parietes of the abdomen and the serous visceral investment, for the relief of internal strangulation of the intestines, is one of great importance, and has of late years much engaged the attention of the profession. An interesting case has been published by Dr. Golding Bird and Mr. Hilton in the last (twelfth) volume of the Medico-Chirurgical Transactions, and although the final result proved unfavourable, still it was successful so far as the operation itself was concerned. On reading the particulars I was struck with its similarity, in many respects, to a case $I$ have kept notes of, which came under my notice a short time ago, and the features of which most worthy of notice are as follow:-

The subject was a female, thirty-six years of age, of spare habit, and the mother of children. On the 19th of October, 1847 , early in the morning, I was first recuested to see her, and $I$ found her lying in bed, on her back, with the knees drawn up; she had been for two days under medical treat. ment, but the more urgent symptoms did not appear till this morning. She said she had formerly, at two or three different periods, suffered from pain in the stomach and bowels, which she attributed to indigestion. When I first saw her, in company with Dr. Taylor, house-surgeon to the dispensary, I found that the bowels had not been relieved for two or three days, although active cathartic remedies with glysters had been freely had recourse to. She had frequent vomiting, and could not retain any nourishment. A dull, heavy pain was present over the abdomen generally, but more below, and on the right side of the umbilicus; the countenance was sunken, the general surface cold and clammy rather than otherwise, and the pulse feeble. There was femoral hernia on the right side, about the size of an egg, which felt quite flaccid and loose without the least tension or pain; and it was very easily reduced without more handling than sufficient to empty the intestine of its contents; the point of the index-finger was freely admitted into the mouth of the sac, satisfactorily showing that no adhesion existed internal to the ring, or that this rupture had anything to do with the obstruction from which she was then suffering. Notwithstanding the use of croton oil pills, cathartic draughts, and the administration of large glysters of gruel, (five pints were passed without any difficulty, which were repeated subsequently, (as well as inflating with air, by means of the enema syringe, the large intestines, no re-actions were effected, except the passing of a little freculent matter from the colon. The vomiting became more agrravated; the symptoms increased in severity; and she died on the evening of the 21st, making nearly three days from $\mathrm{my}$ first visit. There was, notwithstanding the constant sickness, a free secretion of urine; she voided daily to the time of her death about one and a half to two pints.

The post-mortem examination was made about thirty hours after. Abdomen tense and full, from the bowels being distended with air. The peritonæum was inflamed, as was also the omentum; shreds of lymph, and plastic, organizable matter were found agglutinating together some of the convolutions of the small intestines. There were other adhesions of longer standing, having a thread-like character; others again, forming bands varying from three to six inches in length, were broken up in exposing the intestines in their extent. The omentum, adherent to the peritonæum, lining the wall of abdomen in front, and extending below the umbilious by similar adventitious prolongations, was retained to the brim of the pelvis and upper part of the bladder: posteriorly, bands extended between the intestines to the mesentery lumbar region, and upper part of sacrum. Much inflammation was very evident in the ileum, which, for about twelve inches in length, was of a dark claret 
colour. This portion of the tube was about fourteen or six. teen inches from the caput cæcum coli, and was found to be tied down very tightly by one or two bands like a thin cord, exceedingly firm and resisting, which included the above-mentioned knuckle of intestine, and was situated on the right side and below the umbilicus. It appeared as if the bowel had become twisted over these bands, which most effectually closed the canal against the passage of any liquid through it; the middle and internal coats were ulcerated, leaving little more than the peritonæal. 'The intestine within the stricture was much thickened, and that part on the stomach side was inflated with air; but the portion nearer the cæcum was quite empty, and much congested even to the cæcum. The stricture was so tight that it was quite impossible to draw the intestine without laceration; and in order to liberate, it was divided with the scalpel. The internal ring was examined, and found quite free from any intestinal adhesions.

There was no doubt in my mind that the whole mischief resulted from an internal strangulation of some portion of the intestinal canal, (and that opinion was, previously to death, communicated to the friends.) From the free passage and retention for an hour or two of the large injection, as well as the fæculent escape of matter, I was pretty certain that the colon was not implicated, and consequently the obstruction must be in the small intestine. It was, moreover, evident that the femoral hernia had nothing to do with it, as it was never in an irreducible state, the patient being able to return it herself without any difficulty. In the case reported by Dr. Bird and $\mathrm{Mr}$. Hilton, the intestine strictured was the ileum, and about the same distance from the cæcum; the adhesions also were many of them of long standing. The stricture in their case was by a circle formed by another portion of intestine, and maintained in this form by strong adhesions; in the present case, it was solely by strong adventitious membraneous bands. In both cases the intestine on the stomach side was distended, and quite empty on the cæcal; this is of importance in cases when operations are being performed, and enables the surgeon to follow up from the empty part the seat of the obstruction; but even in the dead subject this was not done without some difficulty and delay. The convolutions being distended with air completely hid from view the strictured portion, which was fixed behind, firmly, to the right lumbar region, immediately above the sacrum. The secretion of urine in both cases was abundant; but the particular character of pain, mentioned by them, (Dr. Bird and Mr. Hilton,) by a slight dragging or giving way on the right side, "corresponding to the seat of stricture, was not noticed in the present case. The patient complained of the pain as being "dull and heavy," not lancinating or twisting. It is questionable whether purgatives or even the metallic mercury given, were not rather injurious than otherwise; both would produce inflammation when the stricture was impassable, and the mercury, with an ulcerated condition of the coats, might cause immediate rupture by gravitation. Large and repeated injections of bland liquids appear to be the most feasible. I conceive the above is one of those cases in which an operation might, if performed early, have been attended with success. The point of obstruction was the knuckle of intestine within the stricture, which could only be relieved with the knife; the recent effusion of lymph and the adhesions of the convolutions of intestines were consequent upon this stricture. The bands were no doubt of long standing, and would continue, as previously, to produce little inconvenience, if not interfering with the contents of the bowel, which was not the case, except in the part alluded to above, where they were the result of chronic peritonæal inflammation under which she had suffered, and was mistaken by her for indigestion. There was about one pint and a half of bloody serum in the peritonæal cavity.

Nottingham, April, 1848.

\section{ON THE APPLICATION OF POULTICES.}

\section{By ALFRED MARKWICK, Ese., M.R C.S. \&c., London.}

THE remarks of $M$. Velpeau on poultices, contained in a late LANCET, being, I conceive, somewhat at variance with the now very general opinion of medical men in this country, I am induced, as the introducer of the spongio-piline, to solicit a short space in your journal for the purpose of substantiating the statements I have therein and elsewhere made relative to this material as a means of applying warmtl 1 and moisture.

M. Velpeau states, that "it was believed at one time that poultices were useful merely in virtue of the water they contained, and that if water were kept on the affected part, the same end would be gained as when using a poultice," from which, and from his giving tho preference to linseed-meal, " because it imbibes liquids thoronghly, and contains a certain proportion of very sweet oil, which is not to be found in any other meal," I infer that he considers this opinion to be no longer current, and that the effects produced by these remedies are partly due to the freculent matter of which the body or corpus of the poultice is formed. In my descriptions of the spongio-piline, I have stated, on the authority of several distinguished writers, that warmth and moisture, whether simple or medicated, are the only active ingredients in a poultice, and that the bread or linseed-meal \&c., instead of increasing their soothing and relaxing action, tend, by the irritating qualities they acquire, to destroy them. Thus Dr. Copland remarks, that "fomentations and poultices containing emollient, narcotic, or other medicines, are efficacious, not merely by the moist warmth they afford, but in a great measure by the impression made upon the nervous tissue by the particular medicinal substances they contain;" and that "moist heat employed in various ways is the principal agent by which the physician or surgeon is able to diminish the tension of rigid and unyielding tissues, lessen pressure on sensitive parts, and produce an emollient and soothing effect."* Sir Astley Cooper, after illustrating the sedative effects of heat and moisture by what happens when a person takes a warm bath, observes, "Here, then, is direct proof of what heat and moisture can do when they are applied generally; and when used locally, their action on the part is precisely the same;" + while the late Professor Liston says, that "heat and moisture are the qualities by which a poultice produces its soothing and beneficial effects."

Supposing, then, these views to be correct, it is evident that warmth and moisture should be applied as directly as possible to the skin, in order that they may produce their requisite beneficial action. Can this be done with the ordinary poultice? I maintain that it cannot. Take, for instance, the linseedmeal poultice to which $M$. Velpeau gives the preference, and see what is the condition of the fluid it contains. Is it free or combined? Is it not so intimately blended with, in fact, absorbed, or, as M. Velpeau happily expresses it, imbibed by the meal, as to be prevented frorn coming into this close contact with the cutaneous surface? Can it be done with the spongio-piline? I am prepared to affirm that it can, and for the two following reasons:-1st. It is merely an absorbent, and has no tendency to combine with or clench up, as it were the fluid with which it is impregnated. 2ndly. It is spongy in its texture, and therefore allows the moisture to gravitate towards the surface on which it is applied.

The next.remark to which $I$ wish to refer is as follows:"A poultice first yields its own high temperature to the limb, and afterwards receives heat from it; so that the temperature is always relatively the same. Thus a poultice nay remain twenty-four hours on a limb, and retain a certain amount of heat, except the member be cold," and "as for decomposition it is rare for it to come on before twelve hours, at least, are elapsed, so that changing twice a day is quite sufficient." That a poultice first yields its own elevated temperature to the part upon which it is placed, I am prepared to admit, but I am not prepared to agree with the talented French surgeon in the opinion which, from the above remark, he evidently entertains-viz., that at the end of the twelve hours the poultice possesses the same soothing and relaxing properties as at the commencement. Experience, in fact, tells us to the contrary; moreover, we have in opposition to this the following state ment of the late Professor Liston. He says, "The question has been suggested-How long does a poultice continue to be the bland, inoffensive, and soothing epithem, as when first applied ?" The answer he gives is, "Only for a very short period after its application:" in fact, so prejudiced was he against it, that the very name even of a poultice he considered to be "associated with putrefaction and nastiness."

The spongio-piline, on the contrary, he was well pleased with; he found it to be perfectly free from the slightest tendency to decomposition, besides being clean and comfortable to the patient, retaining its warmth and moisture for a much longer time than any cataplasm.

In making these observations, $I$ wish it to be understood that I am stating nothing but what is fully borne out by the experience of numerous medical men, who have testified to the efficacy of the material in question, and that I am actuated by no other motive than a desire to bring under the notice of the profession, what $I$ believe to be a more convenient and efficacious mode of applying moist heat to the surface of the body.

Langham-place, Aug . 29th, 1848.

* Dictionary, vol. ii. p. 416.

Lectures on Surgery. 